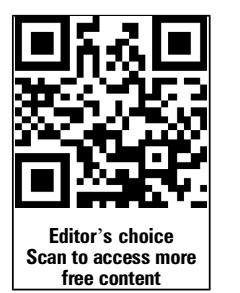

${ }^{1}$ Department of Ophthalmology, Kocaeli University School of Medicine, Kocaeli, Turkey

${ }^{2}$ Department of Ophthalmology, Marmara University School of Medicine, Istanbul, Turkey

${ }^{3}$ Department of Pediatrics, Marmara University School of Medicine, Istanbul, Turkey

\section{Correspondence to} Dr Hande Celiker, Department of Ophthalmology, Marmara Universitesi E. A. Hastanesi, Goz Hastaliklari Anabilim Dali, Pendik, Istanbul, Turkey, drhandeceliker@yahoo.com

Received 8 December 2013 Revised 5 February 2014 Accepted 2 March 2014 Published Online First 21 March 2014
CrossMark

To cite: Karabas $L$, Esen $F$, Celiker $\mathrm{H}$, et al. $\mathrm{Br} \mathrm{J}$ Ophthalmol 2014;98: 1087-1090.

\title{
Decreased subfoveal choroidal thickness and failure of emmetropisation in patients with oculocutaneous albinism
}

\author{
Levent Karabas, ${ }^{1}$ Fehim Esen, ${ }^{2}$ Hande Celiker, ${ }^{2}$ Nursel Elcioglu, ${ }^{3}$ Eren Cerman, ${ }^{2}$ \\ Muhsin Eraslan, ${ }^{2}$ Haluk Kazokoglu, ${ }^{2}$ Ozlem Sahin ${ }^{2}$
}

\begin{abstract}
Purpose The purpose of this work was to describe the choroidal structure in patients with oculocutaneous albinism (OCA).

Methods 20 eyes of 10 patients with OCA and 14 eyes of 7 healthy controls were recruited for the study. Enhanced depth imaging optical coherence tomography (OCT) images of the subjects were taken. The choroidal thickness (CT) was measured from the outer boarder of the retinal pigment epithelium to the inner boarder of sclera at $500 \mu \mathrm{m}$ intervals of a horizontal section from the optic disc. Statistical analysis was performed to evaluate variations in CT at subfoveal and peripapillary
\end{abstract} areas.

Results In the fundoscopic examination and OCT imaging, the foveal light reflex was absent and the foveal pit could not be observed in all of the patients with OCA. The mean subfoveal CT of the patients with OCA was significantly thinner $(242 \pm 56 \mu \mathrm{m})$ compared with healthy controls $(349 \pm 70 \mu \mathrm{m})(p<0.001)$; while there was no statistically significant difference in the peripapillary CTs of the patients with OCA and controls $(157 \pm 42 \mu \mathrm{m}$ vs $151 \pm 31 \mu \mathrm{m}$ respectively, $p=0.77)$, indicating a localised abnormality of choroidal anatomy. Conclusions This study for the first time demonstrated that $\mathrm{CT}$ is decreased in patients with OCA at the subfoveal region. These data combined with the underdevelopment of the foveal pit indicate that there is a generalised developmental or genetic abnormality in the posterior pole of patients with OCA. This choroidal structural abnormality might be related to the previously reported failure of emmetropisation in these children. Further research is needed to understand choroidal metabolism and its relationship with these anatomical changes in albinism.

\section{INTRODUCTION}

Oculocutaneous albinism (OCA) is a condition characterised by the partial or complete absence of pigmentation in the skin, hair and eyes of patients due to a genetic defect in the melanin biosynthesis. Slit lamp examination of these patients reveals transillumination of the iris, ${ }^{1}$ reduced pigmentation of the retinal pigment epithelium (RPE), underdevelopment of the foveal pit and absence of foveal light reflex. $^{2}{ }^{3}$ Misrouting of the optic fibres at chiasm is observed in these patients and this finding can be demonstrated by abnormal visual evoked potential findings. ${ }^{4}$ Other ophthalmological findings commonly observed in these patients include nystagmus and photophobia. ${ }^{5}$ These patients have also been shown to have higher tendency to have refractive errors compared with the healthy population. ${ }^{6}$ As a result of the above-mentioned abnormalities, these patients have decreased visual acuity.

Optical coherence tomography (OCT) is an optical signal acquisition and processing method that is commonly used for retinal imaging. The newer spectral domain OCT imaging technology allows higher resolution and faster imaging. Extended depth imaging (EDI) with spectral domain OCT allows deeper visualisation of the choroidal structure. In EDI-OCT imaging, a spectral domain OCT is used closer to the eye so that an inverted image of the choroid is obtained. Now, newer generation OCT software automatically corrects the inverted image in the choroidal imaging mode. This image allows us to see structural abnormalities in the choroid and also to measure choroidal thickness (CT). ${ }^{7}$

Emmetropisation is a process by which the eye can correct or reduce its own refractive error to produce a clearer image on the retina. Peripheral visual stimuli and scleral metabolism are believed to be important for the emmetropisation process. ${ }^{8}$ Choroid as a vascular tissue between the sclera and retina can act as an accommodative structure by moving the retina forward by thickening and backward by thinning. ${ }^{8}$ Apart from this choroidal accommodation mechanism, growth factors released from the choroid also have the potential to regulate scleral extracellular matrix remodelling, thereby contributing to emmetropisation. ${ }^{8}$

Wildsoet $e t a l^{6}$ studied refractive development in albinism patients and found that emmetropisation is impaired in patients with albinism. Their study also indicated that there was a shift toward hyperopia in the albino patients, but the refractive errors in the group were spread in a broad range from high hyperopia to high myopia. In this study, an axial origin to the refractive errors was supported by high correlation with axial length and refractive errors. In another study, Healey et $a l^{9}$ studied the refractive errors and their correlation with the degree of foveal hypoplasia in patients with nystagmus. They found that foveal hypoplasia did not affect emmetropisation; in the foveal hypoplasia group only the children with albinism had significantly higher refractive errors. They suggested that a structural abnormality other than foveal hypoplasia contributed to the failure in emmetropisation. In this study, we studied whether a change in choroidal structure with EDI-OCT is present in patients with OCA to explain the above-mentioned failure of emmetropisation. 
Table 1 Clinical characteristics of the patients

\begin{tabular}{lll}
\hline & $\begin{array}{l}\text { Patients with OCA } \\
\text { ( } \mathrm{n}=20 \text { eyes) }\end{array}$ & $\begin{array}{l}\text { Controls } \\
(\mathbf{n}=14 \text { eyes) }\end{array}$ \\
\hline Girls/boys & $2 / 8$ & $3 / 5$ \\
Mean age (years) & 10.6 & 9.4 \\
Mean BCVA & $20 / 154$ & $20 / 20$ \\
Transillumination of iris & $20 / 20$ & $0 / 14$ \\
Absence of foveal light reflex & $20 / 20$ & $0 / 14$ \\
Nystagmus & $20 / 20$ & $0 / 14$ \\
Photophobia & $10 / 10$ & $0 / 7$ \\
\hline BCVA, best corrected visual acuity: OCA &
\end{tabular}

\section{MATERIALS AND METHODS}

We included in this study 20 eyes of 10 patients with OCA ( 2 girls and 8 boys, mean age 10.6 years) and 14 eyes of 7 healthy controls ( 3 girls and 4 boys, mean age 9.4 years) (table 1). Three of the patients had a genetic diagnosis OCA 1 and seven patients only had the clinical diagnosis of OCA. Their clinical examination was also consistent with OCA 1. For every subject best corrected visual acuity (BCVA) according to cycloplegic refraction was determined and slit lamp examination and dilated fundus examination were performed. Choroidal imaging was performed with Optovue spectral domain OCT in the EDI mode. The CT was measured from the outer boarder of the RPE to the inner boarder of sclera at $500 \mu \mathrm{m}$ intervals of a horizontal section from the optic disc. Subfoveal (average of 4000,4500 and $5000 \mu \mathrm{m}$ distance from the optic disk measurements) and peripapillary (average of 500 and $1000 \mu \mathrm{m}$ distance from the optic disk measurements) CT were specifically tabulated for statistical analysis. Representative images are given for patients and control subjects in figure 1. All of the measurements were performed by the same observer. Statistical analysis was done using the Mann-Whitney U test.

\section{RESULTS}

In the slit lamp examination, all of the patients with OCA had transillumination of the iris, photophobia and nystagmus, while these findings were not observed in any of the healthy control subjects. Foveal light reflex could not be observed and the choroidal vessels were prominent due to the absence of pigmentation in RPE in all of the patients with OCA, while these findings were normal in the healthy subjects (figure 2). Mean BCVA of the patients with OCA was 20/154 and for the control subjects it was 20/20 (BCVA could not be measured in one patient with OCA due to autism) (table 1).

The mean subfoveal CT of the patients with OCA was significantly thinner $(242 \pm 56 \mu \mathrm{m})$ compared with healthy controls $(349 \pm 70 \mu \mathrm{m})(\mathrm{p}<0.001)$. There was no statistically significant

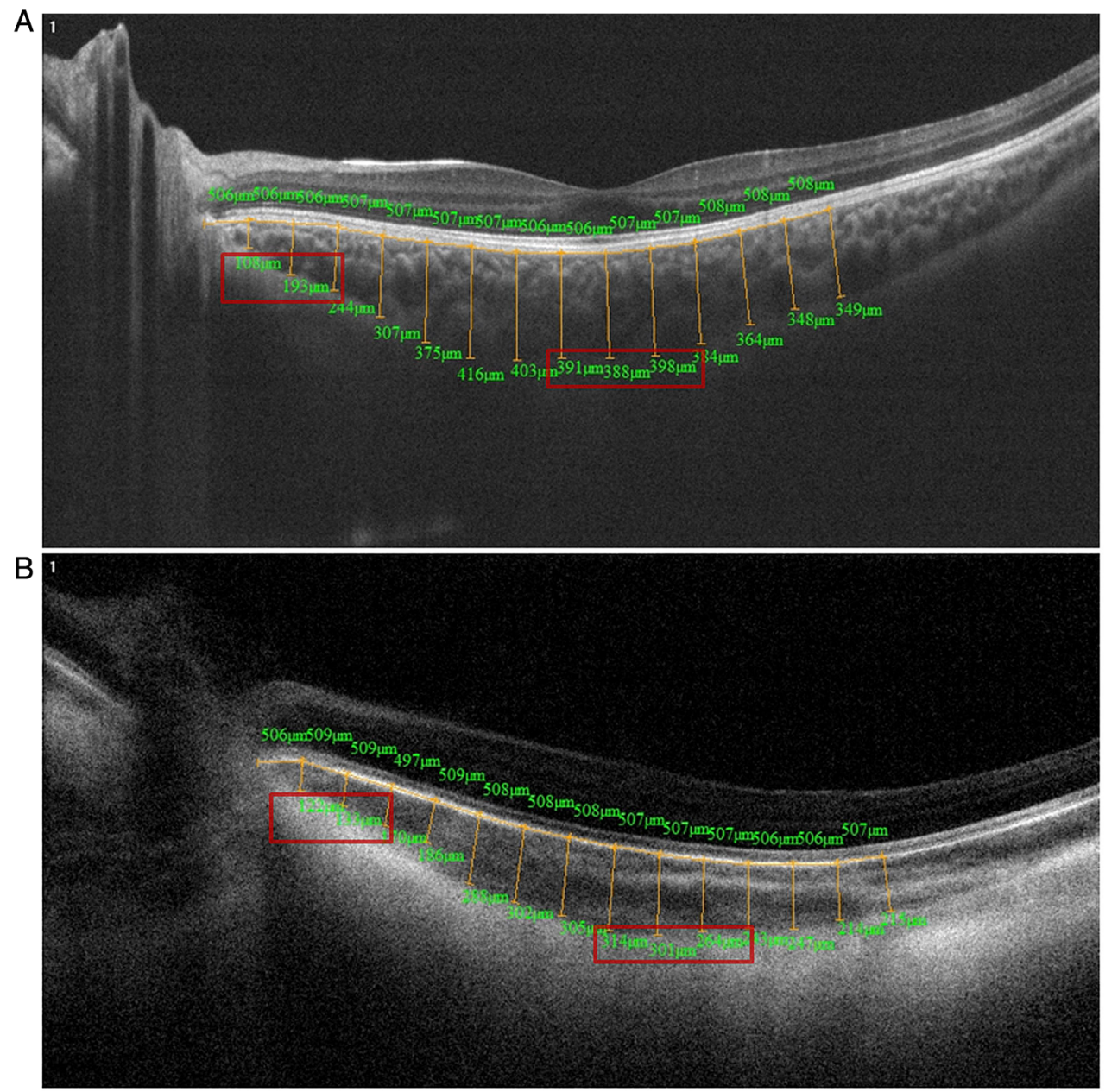

Figure 1 Representative enhanced depth imaging optical coherence tomography (EDI-OCT) images from healthy subjects and patients with oculocutaneous albinism (OCA). (A) This is a representative EDI-OCT image from a control subject. The measurements included in the analysis are marked with rectangles. (B) This is a representative image from a subject with OCA. Here, we also observe the absence of the foveal pit. 
Figure 2 Representative retinal image of a patient with oculocutaneous albinism (OCA). This picture of a patient with OCA demonstrates the hypopigmentation of the retinal pigment epithelium and therefore the choroidal vessels look prominent. We can also observe the absence of foveal light reflex.

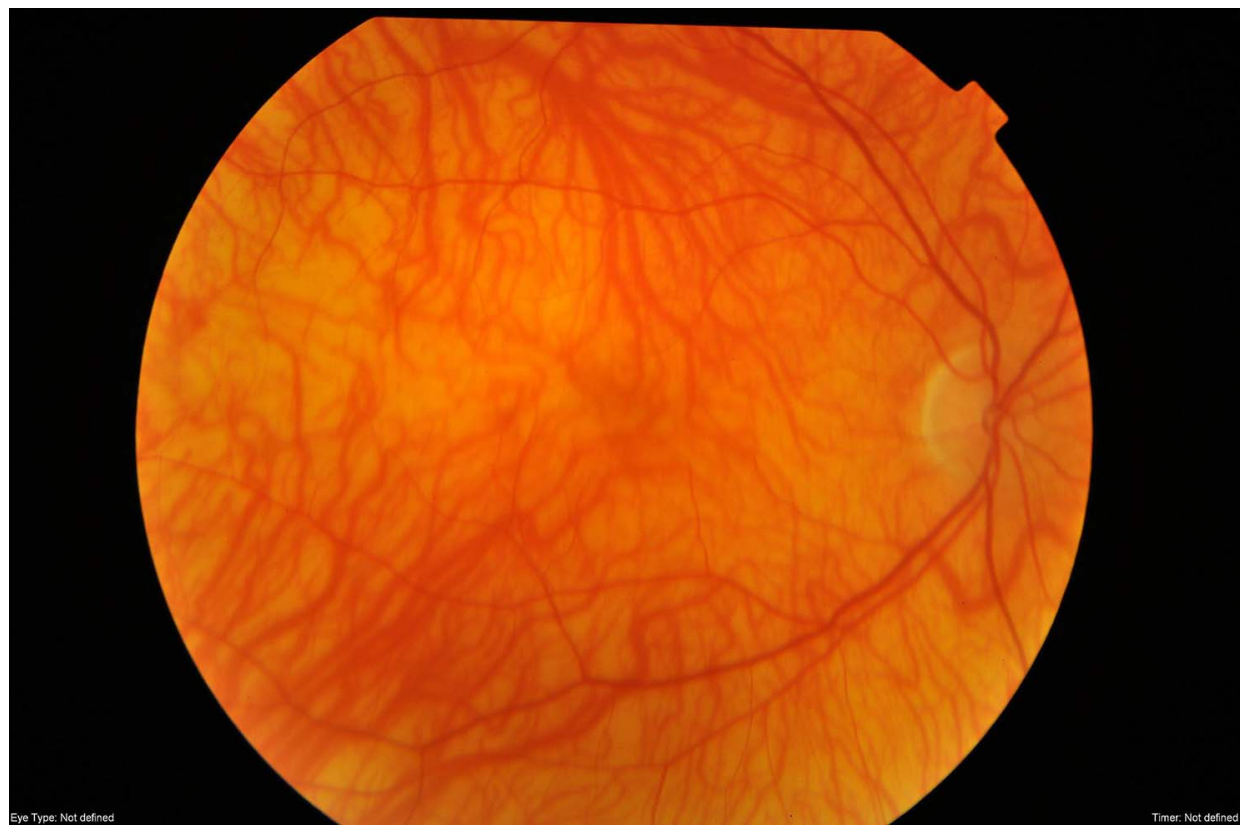

difference in the peripapillary CTs of the patients with OCA and controls $(157 \pm 42 \mu \mathrm{m}$ vs $151 \pm 31 \mu \mathrm{m}$ respectively, $\mathrm{p}=0.77)$. Choroidal thickness values of the patients and controls are given in table 2 .

\section{DISCUSSION}

OCA is a result of a genetic defect in the metabolism of a single pigment, melanin, yet it is associated with abnormalities of various ocular structures. These include hypopigmentation of the iris and RPE, underdevelopment of the foveal pit and misrouting of optic nerve fibres. How a deficiency of a pigment can cause these diverse anatomical changes remains unknown. There is a possibility that some of these diverse anatomical problems are related to abnormalities of vascular support. Until development of EDI-OCT technology, there was no method available that allowed high-resolution imaging of the choroidal structure. In the current study, we have demonstrated for the first time that CT was decreased in patients with OCA under the area where we normally observe the foveal pit in healthy individuals, while it was shown to be normal at other locations in the eye. We believe that the problems observed in the posterior pole of these patients might be related to poor choroidal support or there may be a shared developmental pathway that results in the abnormality of the retinal (foveal pit) and choroidal tissue. We also believe that this choroidal abnormality may be related to the previously described failure of emmetropisation in patients with OCA. ${ }^{6}$

In this study, the mean CT measurements in the healthy control group was slightly higher than the initial descriptive study of choroidal structure in the normal population done by Margolis and Spaide, ${ }^{7}$ while the pattern of CT was similar. CT under the fovea was $287 \pm 76 \mu \mathrm{m}$ in their study while it was 349

Table 2 Refractive status and choroidal thickness measurements of patients with OCA and controls

\begin{tabular}{|c|c|c|c|c|c|c|}
\hline & OD refractive error & OS refractive error & OD subfoveal CT & OD peripapillary CT & OS subfoveal CT & OS peripapillary CT \\
\hline Case 1 & $+0.75(+4.50 @ 96)$ & +2.75 (+4.25@81) & 270 & 93 & 224 & 180 \\
\hline Case 2 & +4.00 (-2.50@2) & +3.75 (-3.00@179) & 307 & 179 & 277 & 193 \\
\hline Case 3 & +8.00 (-2.25@9) & $+7.75(-2.00 @ 10)$ & 216 & 83 & 245 & 157 \\
\hline Case 4 & +8.00 (+1.75@50) & +8.25 (+2.00@75) & 266 & 174 & 293 & 128 \\
\hline Case 5 & $+5.00(-4.50 @ 180)$ & $-5.25(-3.75 @ 175)$ & 240 & 233 & 234 & 148 \\
\hline Case 6 & $-5.25(-4.00 @ 13)$ & $-0.75(-5.25 @ 117)$ & 121 & 124 & 224 & 132 \\
\hline Case 7 & $+0.50(+3.50 \times 107)$ & +1.00 (+3.50@84) & 276 & 120 & 235 & 164 \\
\hline Case 8 & +0.50 (+4.75@96) & +0.00 (+4.25@71) & 348 & 199 & 270 & 228 \\
\hline Case 9 & $+2.50(-3.25 @ 173)$ & +2.50 (+4.25@81) & 159 & 130 & 152 & 117 \\
\hline Case 10 & $+0.00(-2.00 @ 173)$ & $-1.25(-2.50 @ 175)$ & 192 & 169 & 293 & 200 \\
\hline Control 1 & +3.50 (-3.50@180) & +4.50 (-4.25@174) & 350 & 182 & 467 & 182 \\
\hline Control 2 & $+0.00(-0.50 @ 175)$ & $+0.00(-0.75 @ 180)$ & 449 & 206 & 392 & 151 \\
\hline Control 3 & +0.00 & +0.00 & 294 & 98 & 327 & 98 \\
\hline Control 4 & -0.50 & -0.75 & 383 & 171 & 352 & 157 \\
\hline Control 5 & +1.00 (-0.50@5) & $+1.25(-1.00 @ 161)$ & 371 & 151 & 392 & 156 \\
\hline Control 6 & $+2.25(-0.50 @ 12)$ & $+2.25(-0.50 @ 178)$ & 265 & 149 & 207 & 161 \\
\hline Control 7 & $+1.25(-1.50 @ 105)$ & +1.00 (-1.25@73) & 297 & 122 & 333 & 142 \\
\hline
\end{tabular}

CT, choroidal thickness; OCA, oculocutaneous albinism; OD, oculus dexter; OS, oculus sinister. 
$\pm 70 \mu \mathrm{m}$ in our study, and in the nasal region, CT was 145 $\pm 57 \mu \mathrm{m}$ in their study while it was $151 \pm 31 \mu \mathrm{m}$ in our study. This difference probably occurred due to two main reasons. First, the values they reported represented an adult population and our results represented a paediatric population. Their data also demonstrated that CT is higher in the younger population compared with the older population. Second, the OCT instruments used in these two studies were different. Margolis and Spaide $^{7}$ also discussed that CT measurements are quite variable using different instruments and are only meaningful when comparing measurements performed with the same instrument.

In this study, the most important limitation was the low number of subjects. Because OCA is a rare condition, it was hard to find enough patients to expand the study population. We believe that evaluation of different OCA subtypes could also yield more specific results for each disease subgroup, but such an analysis could not be performed due to the low number of participants. Another limitation of this study was that all patients with OCA had nystagmus that made OCT imaging difficult. To overcome this difficulty many images of each eye were taken and the sharpest image was included. One strength of this study was that all examinations and measurements in both groups were done by a single observer to exclude interobserver variability.

This study demonstrated for the first time that the CT of patients with OCA is thinner than that of healthy people. We speculate that this choroidal structural change may be related to the previously shown failure of emmetropisation in these patients. Our hypothesis merely depends on structural data that we could access in the clinical setting. Further research to clarify choroidal metabolism and its relationship with emmetropisation in albinism is warranted to test this yet unproven hypothesis.

Contributors $\mathrm{LK}, \mathrm{FE}, \mathrm{HC}, \mathrm{EC}$ and ME: designed the study, did the study, wrote the manuscript. NE: designed the study, did the study. HK and OS: designed the study, wrote the manuscript.

Competing interests None.

Patient consent Obtained.

Ethics approval Marmara University Ethics Board.

Provenance and peer review Not commissioned; externally peer reviewed.

\section{REFERENCES}

1 Garguilo A, Testa F, Rossi S, et al. Molecular and clinical characterization of albinism in a large cohort of Italian patients. Invest Ophthalmol Vis Sci 2011;52:1281-89.

2 Izquierdo NJ, Emanuelli A, Izquierdo JC, et al. Foveal thickness and macular volume in patients with oculocutaneous albinism. Retina 2007;27:1227-30.

3 Harvey PS, King RA, Summers CG. Spectrum of foveal development in albinism detected with optical coherence tomography. J AAPOS 2006;10:237-42.

4 Von dem Hagen EA. Identifying human albinism: a comparison of VEP and fMRI. Invest Ophthalmol Vis Sci 2008:49:238-49.

5 Gronskov K, Ek J, Brondum-Nielsen K. Oculocutaneous albinism. Orphanet J Rare Dis 2007;2:43-50.

6 Wildsoet CF, Oswald PJ, Clark S. Albinism: its implications for refractive development Invest Ophthalmol Vis Sci 2000;41:1-7.

7 Margolis R, Spaide RF. A pilot study of enhanced depth imaging optical tomography of the choroid in normal eyes. Am J Ophthalmol 2009;14:811-15.

8 Summers JA. The choroid as a sclera growth regulator. Exp Eye Res 2013;114:120-7.

9 Healey N, McLoone E, Mahon G, et al. Investigating the relationship between foveal morphology and refractive error in a population with infantile nystagmus syndrome. Invest Ophthalmol Vis Sci 2013;54:2934-9. 